\title{
LA DIETA CARNICA EN LA COVA DEL BOLOMOR (VALENCIA, ESPAÑA). UNA ESTRATEGIA DE DIVERSIFICACIÓN EN EL PLEISTOCENO MEDIO EUROPEO (MIS 9-5E)
}

\section{MEAT DIET AT BOLOMOR CAVE (VALENCIA, SPAIN). A DIVERSIFICATION STRATEGY IN THE EUROPEAN MIDDLE PLEISTOCENE (MIS 9-5E)}

\author{
Ruth Blasco ${ }^{1}$ y Josep Fernández Peris ${ }^{2}$ \\ 1 The Gibraltar Museum, 18-20 Bomb House Lane, PO Box 939, Gibraltar. \\ E-mail: rblascolopez@gmail.com \\ 2 SIP (Servei d'Investigació Prehistòrica), Museo de Prehistoria, Dip. de Valencia, C/Corona, 36, 46003 \\ Email: josep.fernandez@bolomor.com
}

Presentado el: 19/02/2014 - Aceptado 23/10/2014

\section{Resumen}

La diversificación de la subsistencia -su potencial-en periodos anteriores al Paleolítico superior ha configurado durante los últimos 40 años uno de los principales temas de discusión prehistórica en Europa. En medio de este debate, la Cova del Bolomor, con una cronología de entre 350 y 100 mil años antes del presente, surge como una de las evidencias más claras y antiguas del consumo sistemático de pequeños animales (e.g., lagomorfos, aves, tortugas) por homínidos anteriores a Homo sapiens. Los datos de Bolomor plantean la necesidad de reconsiderar aspectos e interrelacionar factores para explicar la diversificación de esta subsistencia en momentos anteriores al Paleolítico superior. En este estudio, presentamos los datos zooarqueológicos procedentes de tres niveles de la secuencia estratigráfica de Bolomor (XVII, XI y IV) con el objetivo de aportar una visión diacrónica de la dieta cárnica en este enclave. Los datos proporcionados por Bolomor permiten plantear que los cambios en la dieta humana no fueron lineales en el tiempo y en el espacio, sino que probablemente estuvieron condicionados por múltiples factores, en los que el patrón ocupacional, la diversidad del comportamiento y de forma significativa, las características medioambientales locales desempeñaron un papel importante.

Palabras claves: amplio espectro, pequeñas presas, Pleistoceno medio, Cova del Bolomor

\footnotetext{
Abstract

The diversification of subsistence in pre-Upper Paleolithic periods has been the focus of one of the main topics of discussion in Europe over the past 40 years. In the middle of this debate, Bolomor Cave, dated
} 
between 350 and $100 \mathrm{kya} B P$, emerges as one of the clearest and earliest evidence of the systematic use of small prey (e.g., rabbits, birds, tortoises) in to Homo sapiens. Data from Bolomor indicate the need to reconsider aspects and interrelate factors to explain this diversification of subsistence in chronologies prior to the Upper Paleolithic. In this study, we present zooarchaeological data from three levels of the stratigraphic sequence of Bolomor Cave (XVII, XI, and IV) in order to provide a diachronic view of the meat diet at this site. Data provided by Bolomor allow us to establish that the changes in the human diet were not linear in time and space, but seem to have been conditioned by multiple factors-among them, the occupational pattern, behavioral diversity, and local environmental characteristics tahat could have played an important role in the diversification of subsistence.

Keywords: broad spectrum diet, small game, Middle Pleistocene, Bolomor Cave

\section{Introducción}

Los recursos económicos, tanto vegetales como animales, son utilizados por los grupos humanos de acuerdo a sus necesidades alimenticias, técnicas y/o culturales. En la mayoría de las ocasiones, la explotación de estos recursos responde a determinadas circunstancias, las cuales parecen estar principalmente condicionadas por parámetros ecológicos. Dentro del espectro de recursos potencialmente explotables en un paisaje concreto, la obtención de una presa implica la toma de decisiones. Esto es, la captura de ésta conlleva el diseño de una estrategia, la valoración del coste que supone el transporte, la captura y el procesamiento, y el tiempo invertido respecto al retorno tanto energético como social. Luego, el forager puede encontrar una alta diversidad de recursos potencialmente explotables durante sus movimientos por el territorio en busca de alimento, sin embargo éste puede realizar una selección de aquellos que considera adecuados o, por el contrario, puede aprovechar los recursos disponibles sin realizar ninguna discriminación previa. Desde esta perspectiva, la muestra faunística procedente de un yacimiento arqueológico puede reflejar tanto estrategias económicas generales como decisiones puntuales que generan eventos "atípicos" en el conjunto.

La teoría del forrajeo óptimo u optimal foraging theory (OFT) es la base conceptual de análisis que intenta establecer los tipos de condicionantes que existen a la hora de seleccionar los recursos y satisfacer las necesidades alimentarias humanas. La definición de forrajeo engloba las tácticas utilizadas para obtener recursos no producidos, no cultivados y no manipulados por una población humana. Por su parte, el concepto de optimización es el que proporciona una estrategia en la selección de los recursos, desarrollando una direccionalidad evolutiva del comportamiento humano. Para calcular el forrajeo óptimo o la rentabilidad en la subsistencia se tienen en cuenta diversas variables que intervienen en la toma de decisiones (Winterhalder 1981; Simms 1987):

1) el coste y tiempo de búsqueda, que valora el esfuerzo energético y tiempo que supone la exploración de un territorio antes de la captura de una presa. El esfuerzo invertido en la búsqueda depende directamente de la abundancia de presas (o tasa de encuentro), las técnicas utilizadas para su exploración, las características de la presa y los condicionantes del entorno.

2) el coste de persecución, de captura y de preparación de la presa, que valora el tiempo y esfuerzo invertido durante el transcurso de estos procesos. 
En base a estos parámetros, la relación entre el radio de encuentro y de handling time (coste/esfuerzo de la persecución, captura y preparación de la presa) podría establecer el grado de eficiencia en la obtención de recursos, permitiendo realizar predicciones sobre la viabilidad de su explotación. Según estas predicciones, los recursos serían adquiridos según su orden de eficiencia y por ello, los animales de alto rango son los que producirían una ganancia energética en relación al esfuerzo realizado. En todos estos estudios, los animales del tipo lepóridos o aves no alcanzan una posición destacable como especie de alta rentabilidad, ya que su pequeño tamaño podría implicar un gasto energético elevado en su búsqueda, captura y procesamiento en relación al retorno energético que ofrecen.

Dentro de los modelos de la "Teoría del Forrajeo Óptimo", la amplitud de la dieta (Diet-breadth model) también aborda el tema de la selección de los recursos dentro de un entorno concreto. La discusión de este modelo comienza cuando Binford (1968) observa una diversificación importante en la dieta humana, caracterizada principalmente por la inclusión de nuevas especies, al final del Paleolítico en la latitud media y alta de Europa. Para dar una explicación a este cambio en la explotación de los recursos, se propusieron diferentes hipótesis interconectadas basadas en la "Revolución de Amplio Espectro" (Broad Spectrum Revolution -BSR) (Flannery, 1969). En 1999, Stiner et al. publicaron un modelo para explicar, entre otras cosas, la aparente contradicción entre la explotación intensiva de pequeñas presas, y más concretamente lagomorfos, y la teoría del forrajeo óptimo. Este artículo no fue unánime y a partir del cual, surgió una importante red de debates que tuvieron como base fundamental dar respuesta a esta amplitud dietética así como determinar el origen de la explotación intensiva de pequeños animales (e.g., Stiner et al. 2000; Stiner 2001; Stiner y Munro 2002; Bar-El y Tchernov 2001; Aura et al. 2002; Hockett y Haws 2002; Byers y Broughton 2004). De forma general, se han propuesto diversas hipótesis vinculadas tanto a la presión ambiental, como a la presión cinegética y tecnológica. Sin embargo, estos factores parten de modelos teóricos y predictivos sobre el comportamiento humano y el entorno, siendo necesario evaluar la existencia de variables no controladas que también podrían haber provocado, al menos en algunas ocasiones, la incorporación de ciertos animales a la dieta. Éstas además no tendrían porqué estar siempre condicionadas por la necesidad de optimizar los recursos, sino que podrían estar inmersas en el marco de la "ecología nutricional" (Hockett y Haws 2002, 2009; Haws 2003; Hockett 2006, 2007) y/o responder a normas culturales o relaciones sociales, las cuales no aportarían a priori ningún beneficio económico sino por el contrario, algún tipo de reconocimiento cultural o social Alhaique 1994; Bar-El y Tchernov 2001; Fontana 2003; Lupo y Schmitt 2005; Wadley 2010).

Partiendo de un enfoque nutricional, Hockett y Bicho (2000) calculan algunos parámetros, como por ejemplo la médula contenida en los elementos apendiculares de Lepus granatensis y Oryctolagus cuniculus, la cual muestra unas proporciones de aproximadamente $7 \mathrm{~g}$ de grasa para la liebre, y $3 \mathrm{~g}$ para el conejo. Estos investigadores también advierten sobre la alta tasa de niacina (vitamina B3), fósforo y potasio presente en estos animales. Estos componentes son importantes para el crecimiento y correcto desarrollo metabólico del individuo, lo cual podría llevar a la búsqueda consciente o inconsciente de estos recursos, más allá del esfuerzo o tiempo que conlleve su obtención y procesamiento.

Por otro lado, existen muchos ejemplos etnológicos y arqueológicos que documentan la importancia de los recursos no comestibles tanto de lepóridos como de aves o quelonios. Algunos ejemplos son la utilización de huesos apendiculares de lepóridos como agujas (e.g. Honeisen y Peyer 1994; Leesch 1997) o como ornamentos (Hockett 1994; Laroulandie y 
D'Errico 2004), el aprovechamiento de plumas de diferentes tipos de rapaces o garras con fines simbólicos (Mourer-Chauviré 1989; Fiore et al. 2004; Soressi et al. 2008; Gaudzinski y Niven 2009; Peresanni et al. 2011; Finalyson et al. 2012; Morin y Laurandie 2012; Romandini et al. 2014) y el uso de los caparazones de tortuga como posibles contenedores (Speth y Tchernov 2002) o instrumentos musicales (Acuña 1982-1987). Incluso en términos de rentabilidad energética, se debe tener en cuenta que las especies de movimiento lento, como las tortugas, podrían relacionarse con una alta tasa de retorno si existiera la posibilidad de que éstos fueran obtenidos por algunos miembros del grupo que presentasen una movilidad reducida (Morín 2012) -i.e., la inclusión de algunos pequeños animales a la dieta podría convertirse en rentable a partir de la existencia de un reparto de tareas según las características individuales de los integrantes del grupo, como edad, sexo y/o limitaciones físico-motrices temporales o permanentes.

Este artículo pretende aportar datos zooarqueológicos procedentes de la Cova del Bolomor que contribuyan al debate sobre el consumo de pequeños animales y al conocimiento de los modos de vida de los grupos humanos del Pleistoceno medio europeo.

\section{Cova del Bolomor}

Cova del Bolomor se sitúa a $2 \mathrm{~km}$ al SE de la población de Tavernes de la Valldigna (Valencia, España) y a una altitud de $100 \mathrm{~m}$. Esta ubicación corresponde a la estribación septentrional del Macizo del Mondúver y vertiente meridional de la depresión de La Valldigna, una subcomarca de la provincia de Valencia que configura el límite estructural geológico entre los dominios Ibérico y Bético (Figura 1).

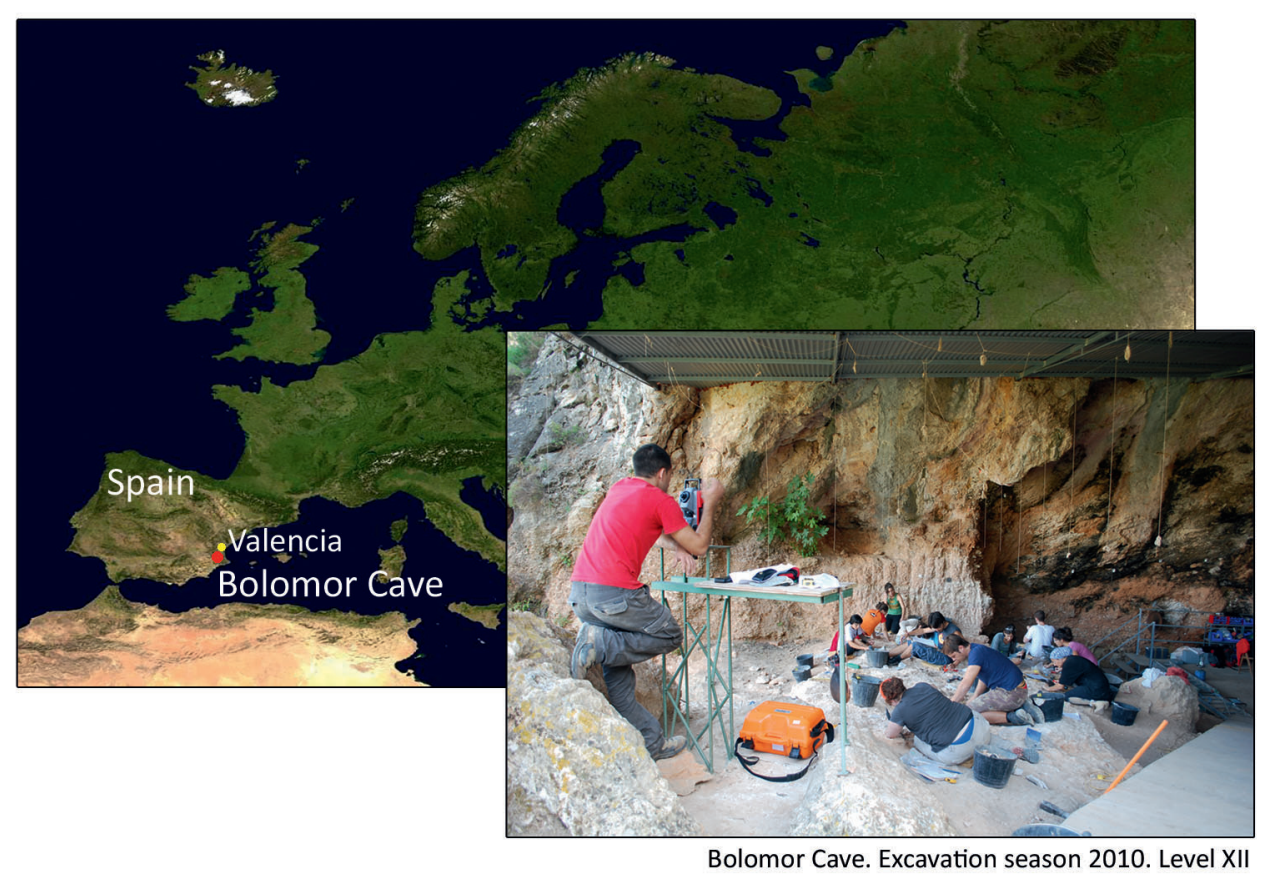

Figura 1. Localización de la Cova del Bolomor, Valencia, España. 
El relleno sedimentario de Cova del Bolomor está formado en su mayor parte por material alóctono de origen coluvial. Estos aportes forman una serie sedimentaria que se apoya en el roquedo cretácico y se inicia con potentes niveles estalagmíticos. Estos ocupan todo el suelo de la cavidad, desde la entrada hasta el punto más interior con coladas adaptadas a una topografía irregular y con formaciones procedentes de goteos cenitales. Sobre este primer relleno (nivel XVIII) se apilan materiales con proyección subhorizontal y potencia variable según el lugar entre 4 y $10 \mathrm{~m}$. La estratigrafía de este cuerpo sedimentario detrítico sería en síntesis, de techo a muro, en el sector oeste de referencia para todo el yacimiento, la siguiente (Fumanal 1993) (Figura 2):

- Fase Bolomor I (niveles XVII a XV). Ciclo climático fresco de cierta humedad que registra una brechificación del sedimento. En el nivel XVII se ha obtenido una datación por racemización (aminoácidos) de esmalte dentario de 525.000 \pm 125.000 años BP.

- Fase Bolomor II (niveles XIV y XIII). Ciclo climático con rasgos interestadiales, templados-cálidos que registra el encharcamiento periódico de la cueva. En el nivel XIV se han obtenido por termoluminiscencia dos dataciones con valores de $233.000 \pm 35.000 \mathrm{y}$ $225.000 \pm 34.000$ años y en el nivel XIII de 152.000 \pm 23.000 años BP. Se inscribe dentro del estadio isotópico 7.

- Fase Bolomor III (niveles XII a VIII). Ciclo climático que evoluciona hacia una situación rigurosa y árida (nivel XII). Corresponde al estadio isotópico 6.

- Fase Bolomor IV (niveles VII a I). Representa las oscilaciones templadas y húmedas del último interglaciar. La elevada humedad ha provocado la inundación parcial de la cueva y la brechificación de los sedimentos. En el nivel II se ha obtenido una datación absoluta por TL de121.000 \pm 18.000 años BP. Esta fase se relaciona con el estadio isotópico 5e.

Respecto al conjunto faunístico, Cova del Bolomor ha documentado un total de treinta especies animales, entre las que se incluyen primates, carnívoros, herbívoros, pequeñas presas (conejos, aves y tortugas) e incluso, de forma puntual restos de peces. Los animales que tienen una mayor representación a lo largo de su secuencia estratigráfica son el ciervo (Cervus elaphus) y el caballo (Equus ferus), con representación relativamente inferior de gamo (Dama sp.), tar (Hemitragus bonali y Hemitragus cedrensis en el nivel IV), uro (Bos primigenius), megaloceros (Megaloceros giganteus), asno silvestre (Equus hydruntinus), rinoceronte de estepa (Stephanorhinus hemitoechus), jabalí (Sus scrofa), macaco (Macaca sylvana), elefante (Paleoloxodon antiquus), hipopótamo (Hippopotamus anphibius), entre otros. Los porcentajes de pequeños animales, especialmente del conejo en el nivel IV y las anátidas en el nivel XI superan conjuntamente en algunos casos el 50\% del número mínimo total de individuos (Blasco y Fernández Peris 2009, 2012a, 2012b; Blasco et al. 2013a). La presencia de carnívoros es esporádica, no obstante, se han recuperado restos de Ursus arctos, Ursus tibetanus, Canis lupus, Panthera leo, Lynx pardina, Vulpes vulpes y Meles meles (Blasco 2011).

Actualmente, la industria lítica recuperada en la Cova del Bolomor está compuesta por más de 50.000 piezas líticas, de las cuales sólo un $11 \%$ son artefactos retocados (Fernández Peris 2007; Fernández Peris et al. 2008). Las materias primas más utilizadas son el sílex, la caliza y la cuarcita que proceden de cantos marinos, coluviales y fluviales del entorno más inmediato al enclave, pero también se han localizado afloramientos más alejados (aproximadamente a $15 \mathrm{Km}$. del yacimiento) en las cuencas del Xùquer y del Serpis. La industria lítica de Bolomor puede considerarse como un conjunto o tecno-complejo de lascas pertenecientes al Paleolítico medio temprano. La variabilidad observada a lo largo de la estratigrafía escasazo es altamente significativa y parece estar marcada por las características 


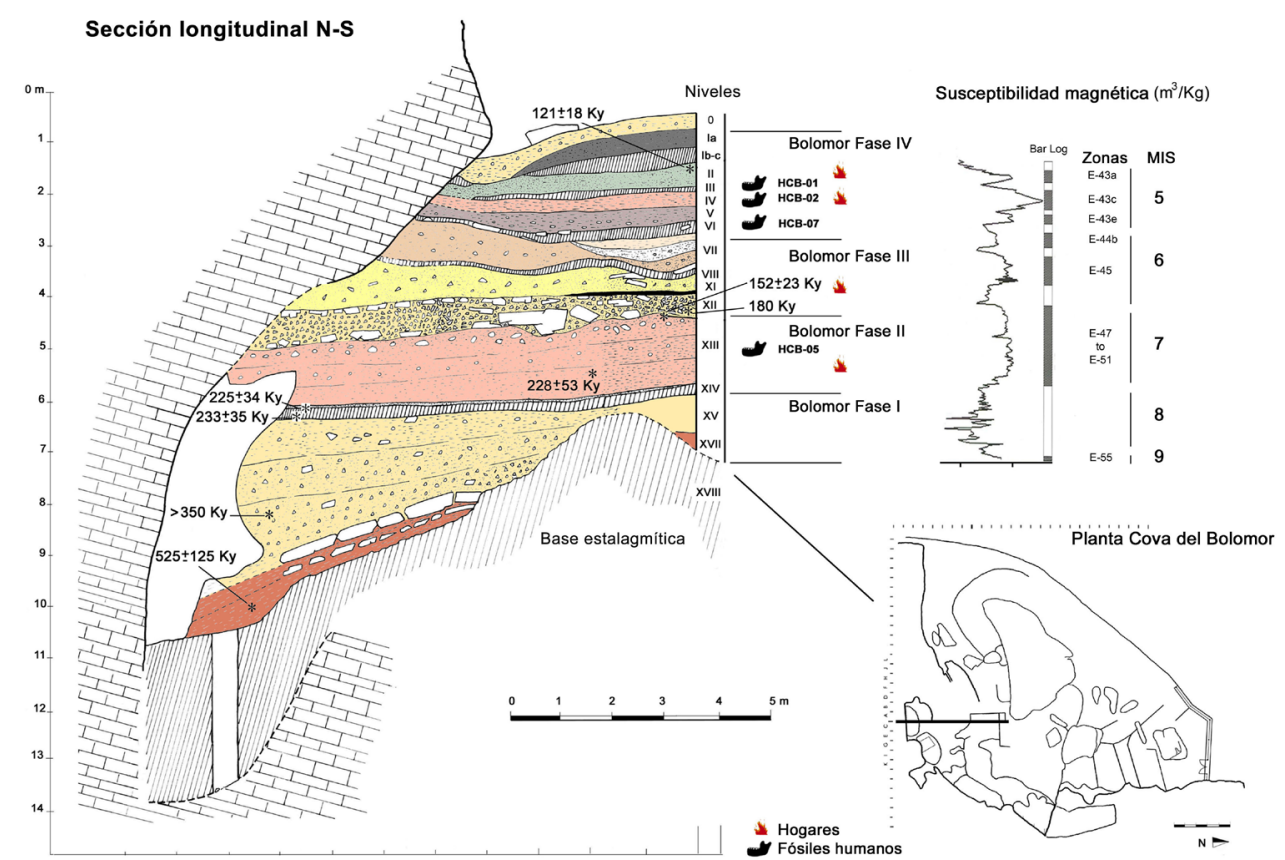

Figura 2. Secuencia estratigráfica de la Cova del Bolomor, Valencia, España.

ocupacionales que los grupos humanos realizan durante la formación de cada nivel. A pesar de ello, los conjuntos líticos parecen adquirir cierta complejidad en los momentos más recientes de la secuencia, coincidiendo con una mayor intensidad en las ocupaciones humanas (Fernández Peris 2007). Las características tecnológicas apuntan a una industria lítica no levallois, no facetada y no laminar, formada por un amplio repertorio de utillaje sobre lasca altamente retocado. No se han recuperado bifaces, hendedores, triedros o cantos tallados en toda la secuencia estratigráfica. Según los estudios desarrollados por Fernández Peris (2007), el registro lítico de Bolomor se caracteriza por ser un tecno-complejo de lascas de pequeño formato (con microlitismo no laminar) y un predominio de raederas, denticulados y retoque diversificado. Además, los artefactos suelen mostrar una intensa reutilización especialmente acusada en los niveles superiores de la secuencia (Fernández Peris 2007; Cuartero 2008). El macroutillaje sólo se observa puntualmente en algunos niveles (e.g., nivel XII) a partir de amplios formatos de lascas elaboradas con caliza, sin retocar o con retoque sencillo.

Hasta el momento, se han recuperado restos de la utilización del fuego en los niveles II, IV, XI y XIII (Fernández Peris et al. 2012). En el nivel II, se documentaron posibles vaciados de cenizas como consecuencia de la limpieza de las estructuras de combustión para la preparación de nuevos hogares. En el nivel IV, se documentaron 4 hogares que dejaron como testigo sedimento termoalterado de color rojizo, incluyendo piedras térmicamente alteradas en la base de uno de ellos. El nivel XI documenta 7 hogares simples sin estructuración interna que parecen corresponder a combustiones de corta duración según los análisis y reproducciones experimentales en curso (Fernández Peris et al. 2007). Por último, en el nivel XIIIc se ha registrado la presencia de dos hogares con una estructuración interna clara basada en una preparación del área. Se ha realizado una datación por RA sobre 
malacofauna recogida del área circundante a los hogares de $228 \pm 53 \mathrm{kya}$. Esta fecha indicaría la existencia (por el momento) más antigua del uso controlado del fuego en la Península Ibérica y Sur de Europa.

Los fósiles humanos recuperados hasta ahora en la Cova del Bolomor corresponden a siete piezas óseas y dentales. Algunos de los restos fueron recuperado en las tareas de cribado de los sedimentos removidos por los antiguos trabajos de cantería de los años'30 del siglo pasado y otros han sido recuperados en proceso de excavación y por tanto, con clara ubicación estratigráfica. Según Arsuaga y colaboradores (2012), la morfología de los restos humanos de la Cova del Bolomor es compatible con la de los fósiles humanos europeos del Pleistoceno medio.

\section{Metodología}

Los métodos de análisis han seguido los estándares publicados para la Tafonomía, con especial atención a las alteraciones producidas durante la fase nutricional de la explotación de los animales (e.g., Blasco et al. 2013a; Lyman 2008).

En ocasiones, la fragmentación ósea dificulta la atribución anatómica y taxonómica de los restos faunísticos que componen la muestra. Sin embargo, estos fragmentos a priori "no identificados" han sido clasificados a nivel esquelético según sus características morfológicas en: 1) huesos largos, 2) planos y, 3) articulares (carpales, tarsales y patellas); y a nivel taxonómico en categorías estimadas de peso que dependen del tamaño y la edad de los animales (e.g., ver Blasco et al. 2013a): 1) talla muy grande (taxones mayores de $1500 \mathrm{~kg}$ ), 2) talla grande (entre 300 y $1500 \mathrm{~kg}$ ), 3) talla media (entre 100 y $300 \mathrm{~kg}$ ), 4) talla pequeña (entre 20 y $100 \mathrm{~kg}$ ) y, 5) talla muy pequeña (inferiores a $20 \mathrm{~kg}$ ). La edad de muerte de los animales ha sido estimada a partir del reemplazamiento y desgaste dental (e.g., Silver 1969; Azorit et al. 2002), aunque en ocasiones también se ha recurrido al grado de epifisación de los huesos o al tipo de tejido cortical (compacto en adultos o más poroso en inmaduros).

Tras la identificación anatómica y taxonómica del registro faunístico, el siguiente paso es la contabilización de los restos analizados. Para ello se han utilizado índices como el Número de Restos (NR) o Número de Especimenes Identificados (NISP) y el Número Mínimo de Elementos (MNE). El Número Mínimo de Individuos (NMI) se ha calculado a partir de la repetición de partes anatómicas teniendo en cuenta el lado que ocupa el hueso en el esqueleto (derecho o izquierdo) y la edad del individuo. Estos indicadores permiten establecer el las frecuencias de unidades anatómicas mínimas (MAU). Éste es un porcentaje de representación entre los elementos esqueléticos existentes en el conjunto y los que cabría esperar según los individuos cuantificados. Con el objetivo de valorar y estimar las proporciones entre pequeñas presas y macro-fauna, se ha utilizado el NMI y no el NR o NISP para evitar la distorsión que pueda producir el alto grado de fragmentación presente en el conjunto estudiado.

Los daños óseos generados por los agentes biológicos (principalmente homínidos y carnívoros) han sido tratados tanto a nivel macroscópico como microscópico (lupa binocular -magnification to 120X; microscopio electrónico de barrido ambiental -ESEM, FEI QUANTA 600). Respecto a las alteraciones antropogénicas, se ha prestado especial atención a las marcas de corte, las cuales han sido agrupadas en incisiones, aserrados, tajos y raspados (e.g., Shipman y Rose 1983; Lyman 2008). En los casos en los que la identificación del hueso ha 
sido posible, también se han recogido datos como la posición, la orientación y la disposición de las marcas sobre el elemento esquelético en concreto. En cuanto a los daños generados por los carnívoros, las marcas de dientes se ha agrupado en surcos, depresiones, improntas y vaciados de las epífisis siguiendo a Maguire et al. (1980), Binford (1981) y Blumenschine y Selvaggio (1988).

La fracturación ósea se ha analizado en busca de criterios que puedan indicar cierta estandarización. Por ello, se han situado los impactos de percusión, estigmas y contragolpes sobre los huesos según sus caras y porciones (Blasco et al. 2013b). También se han registrado otros elementos diagnósticos de fracturación intencional, como conos de percusión, lascas medulares, esquirlas parásitas y lascas corticales (e.g., Capaldo y Blumenschine 1994; Pickering y Egeland 2006). Las modificaciones óseas resultantes de la termoalteración se han analizado en términos de presencia/ausencia y basados principalmente en cambios de coloración y otras alteraciones físicas, como fisuras, fracturas o craquelados (e.g., Shipman et al. 1984; Stiner et al. 1995). El grado de alteración se ha designado de acuerdo a 6 categorías de intensidad, siendo el grado 0 los huesos no quemados y el 5 los calcinados. Como en el caso del resto de alteraciones, también se ha registrado el área y región anatómica de la cremación.

\section{Resultados}

El conjunto faunístico de Bolomor procedente de los niveles XVII (XVIIa y XVIIc), XI y IV muestra una alta diversidad específica con más de 30 taxones pertenecientes a las categorías de Cercopithecinae, Carnivora, Ungulata y pequeños animales como Leporidae, Aves, Testudinidae, Amphibia y Salmonidae (Blasco y Fernández Peris 2012a; Blasco et al. 2013a). Cervus elaphus y Oryctolagus cuniculus son los taxones más abundantes con Aythya sp. en el nivel XI y Testudo hermanni en el nivel IV (Tabla 1). Con respecto a la edad de muerte de los animales, existe un grupo claramente predominante sobre el resto. Los individuos adultos son los más representados en todas las especies y tallas de peso (XVIIc $=23 / 30 \mathrm{o}$ $76.7 \%$; XVIIa= 35/38 o 92.1\%; XI= 24/30 o 80\%; IV= 83/90 o 83.8\%) (ver Blasco et al. $2013 \mathrm{a}$ para más detalle).

Las frecuencias de unidades anatómicas mínimas (\%MAU) de los conjuntos estudiados valoran la proporción entre los elementos recuperados y los que cabría esperar según el NMI. De este modo, la representación anatómica obtenida es válida para observar los elementos ausentes en el conjunto. Según este índice, se observa una representación esquelética sesgada en todos los taxones del conjunto. Esta selección de elementos se caracteriza principalmente por la presencia del esqueleto apendicular proximal (estilopodios y zeugopodios) y del craneal (fundamentalmente mandíbulas y maxilares). Por el contrario, las bajas proporciones de basipodios y la casi total ausencia del esqueleto axial y de falanges caracterizan a la mayoría de las especies documentadas en la secuencia (Figura 3). Los elementos con mayor valor nutricional y medular son los principalmente representados en tallas superiores a aproximadamente $20 \mathrm{~kg}$ (Binford 1981; Emerson 1993). Sin embargo, Oryctolagus cuniculus parece funcionar de un modo distinto dentro de los conjuntos siguiendo una tendencia más igualitaria entre sus partes anatómicas, aunque parece existir un cierto predominio de las extremidades superiores e inferiores en el nivel IV, junto a las cinturas en el nivel XI y la pelvis en el XVII. En el caso de las aves, los elementos con más alto índice de supervivencia es el coracoides y el tibiotarso en el nivel XI y IV, y ulnas, húmeros y carpo-metacarpos en el subnivel XVIIa (Tabla 2). 


\begin{tabular}{|c|c|c|c|c|c|c|c|c|c|c|c|c|}
\hline \multirow[t]{2}{*}{ Taxa* } & \multicolumn{3}{|c|}{ XVIIa } & \multicolumn{3}{|c|}{ XVIIc } & \multicolumn{3}{|l|}{ IV } & \multicolumn{3}{|l|}{ XI } \\
\hline & NR & MNE & MNI & NR & MNE & MNI & NR & MNE & MNI & NR & MNE & MNI \\
\hline Macaca sulvana & & & & & & & 1 & 1 & 1 & & & \\
\hline Carnivora indet. & & & & & & & 5 & 4 & & & & \\
\hline Ursus arctos & & & & & & & 1 & 1 & 1 & & & \\
\hline Canis cf. lupus & 4 & 4 & 1 & & & & 2 & 2 & 1 & & & \\
\hline Vulpes vulpes & & & & & & & 2 & 2 & 1 & & & \\
\hline Panthera leo & & & & & & & 3 & 2 & 2 & & & \\
\hline Lunx pardinus & & & & & & & 2 & 2 & 1 & & & \\
\hline Castor fiber & & & & & & & & & & 2 & 1 & 1 \\
\hline P. antiauus & 2 & 1 & 1 & 2 & 1 & 1 & 4 & 2 & 1 & & & \\
\hline S.hemitoechus & 8 & 3 & 2 & 1 & 1 & 1 & & & & 3 & 2 & 2 \\
\hline Eauus ferus & 77 & 30 & 2 & 56 & 22 & 1 & 65 & 25 & 4 & 2 & 2 & 1 \\
\hline E. hudruntinus & 10 & 9 & 1 & 8 & 5 & 1 & 16 & 9 & 1 & & & \\
\hline H. amphibius & & & & & & & 46 & 5 & 2 & & & \\
\hline Sus scrofa & 27 & 20 & 1 & 13 & 10 & 1 & 115 & 55 & 5 & & & \\
\hline M. giganteus & & & & & & & & & & 2 & 2 & 1 \\
\hline Dama sv. & & & & & & & 91 & 41 & 3 & 4 & 4 & 1 \\
\hline Cerous elavhus & 177 & 58 & 4 & 132 & 47 & 4 & 647 & 193 & 12 & 55 & 35 & 4 \\
\hline B. primigenius & 24 & 13 & 1 & 22 & 13 & 1 & 213 & 63 & 4 & 2 & 2 & 1 \\
\hline H. bonali & 28 & 20 & 1 & 6 & 6 & 2 & & & & 16 & 13 & 2 \\
\hline H. cedrensis & & & & & & & 121 & 47 & 3 & & & \\
\hline O. cuniculus & 620 & 346 & 15 & 457 & 234 & 12 & 789 & 440 & 20 & 262 & 150 & 7 \\
\hline Lepus sp. & 3 & 3 & 1 & & & & & & & & & \\
\hline Passeriformes & 5 & 5 & 1 & 9 & 9 & 2 & 25 & 21 & 2 & & & \\
\hline Galliformes & 8 & 7 & 2 & & & & 19 & 16 & 1 & & & \\
\hline Phasianidae & 18 & 14 & 3 & 10 & 9 & 2 & 24 & 16 & 2 & & & \\
\hline Anatidae & 4 & 4 & 1 & & & & & & & & & \\
\hline Anas sp. & & & & 16 & 14 & 2 & 29 & 25 & 2 & & & \\
\hline Authua sp. & & & & & & & 34 & 28 & 3 & 202 & 167 & 8 \\
\hline Corvidae & & & & & & & 20 & 13 & 1 & & & \\
\hline Purrhocorax sp. & & & & & & & 6 & 6 & 1 & & & \\
\hline Columba sp. & & & & & & & 34 & 25 & 2 & & & \\
\hline Strigidae & & & & & & & 1 & 1 & 1 & & & \\
\hline Aves indet. & & & & & & & 17 & 2 & & & & \\
\hline T. hermanni & & & & & & & 526 & 131 & 19 & 4 & 3 & 1 \\
\hline Bufo sp. & 1 & 1 & 1 & & & & 4 & 2 & 2 & & & \\
\hline Pisces & & & & & & & 2 & 2 & 1 & 1 & 1 & 1 \\
\hline T. muv grande & & & & 8 & 1 & & 37 & 6 & & & & \\
\hline T. grande & 186 & 11 & & 219 & 11 & & 1975 & 49 & & 16 & 4 & \\
\hline T. media & 364 & 22 & & 235 & 16 & & 10274 & 116 & & 128 & 14 & \\
\hline T. pequeña & 160 & 24 & & 95 & 11 & & 9053 & 275 & & 247 & 20 & \\
\hline T. muv pequeña & & & & 8 & 1 & & 304 & 61 & & 92 & 8 & \\
\hline Indet. & 6 & & & 10 & & & 816 & & & 9 & & \\
\hline Total & 1732 & 595 & 38 & 1307 & 411 & 30 & 25323 & 1689 & 99 & 1047 & 428 & 30 \\
\hline
\end{tabular}

Tabla 1. NR, NME y NMI procedente de la Cova del Bolomor (Valencia, España). *Los fósiles humanos no han sido incluidos.

A partir del análisis tafonómico de los huesos se observa que homínidos y carnívoros son los agentes biológicos que inciden con mayor frecuencia en la Cova del Bolomor. No obstante, se debe matizar que la proporción de las alteraciones generadas por ambos predadores muestran una intervención antrópica predominante, la cual se manifiesta principalmente por la presencia de marcas de corte y elementos diagnósticos de una fracturación intencional. Respecto a las marcas de corte, se han identificado 119 restos con incisiones en el subnivel XVIIc (9.1\%), 117 en el XVIIa (6.7\%), 79 en el nivel XI (7.5\%) y 1817 en el IV (7.2\%) (Figura 4). Este hecho nos ha permitido, en algunos casos, identificar actividades específicas 


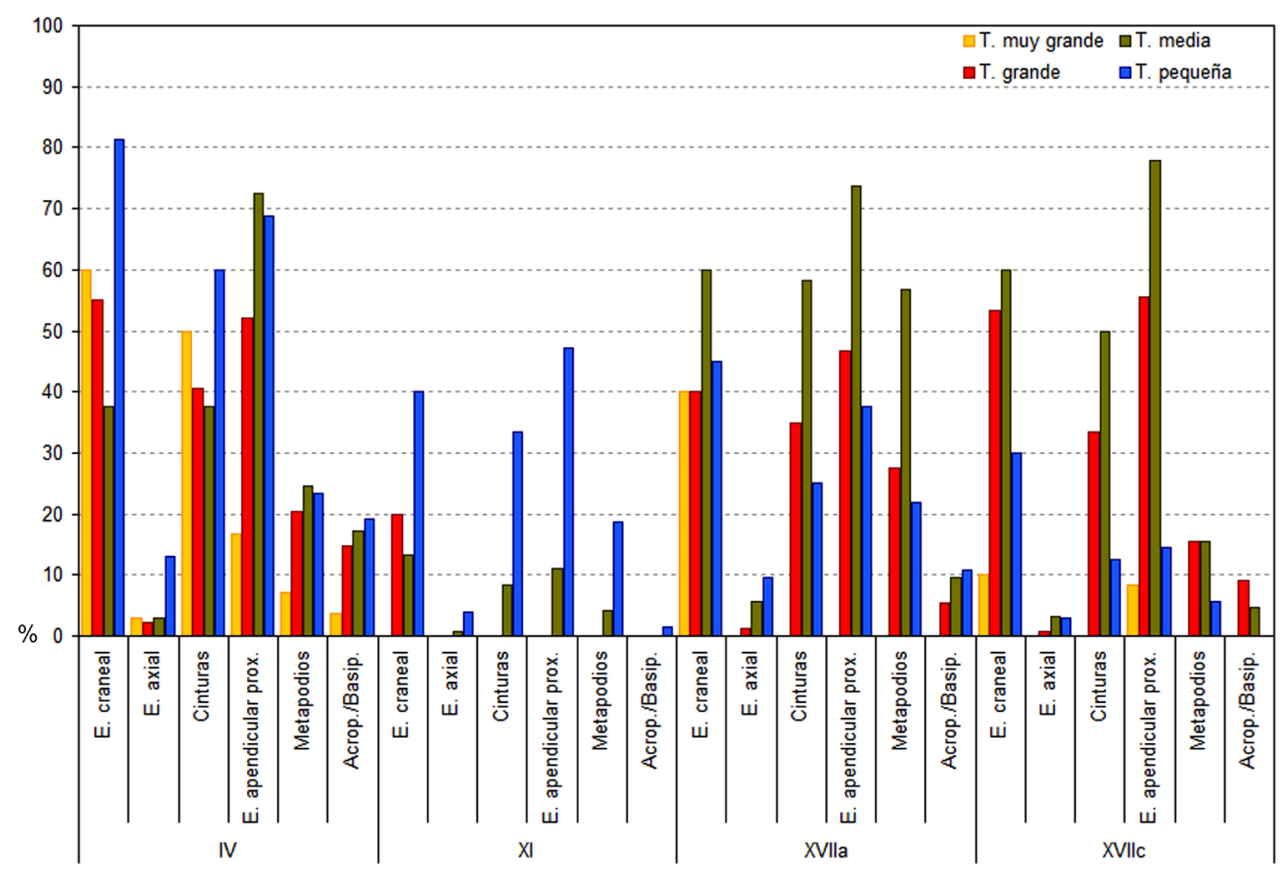

Figura 3. \%MAU de la Cova del Bolomor por niveles y categorías por talla de peso (superiores a $20 \mathrm{~kg})$.

relacionadas con la fase nutricional de la carcasa. En primer lugar, el despellejamiento se ha registrado en partes anatómicas como el nasal, las mandíbulas, los metápodos y las falanges. La evisceración, aunque presente, ha dejado escasas evidencias sobre el conjunto debido por un lado, a la baja representación del esqueleto axial en todos los niveles y por otro, a la frecuente utilización directa de las manos durante su transcurso. Por lo que respecta a la descarnación, podemos afirmar que es la actividad que mayor registro ha proporcionado, identificándose de forma mayoritaria en los huesos largos de las tallas medias y grandes con un predominio de las incisiones en las diáfisis y los aserrados en las metáfisis. En el caso de los animales más pequeños, se han identificado marcas de corte sobre restos de lagomorfos $($ XVIIc $=23 / 457$ o $5 \%$; XVIIa $=14 / 620$ o $2.3 \%$; XI=28/262 o $10.7 \%$; IV=111/789 o $14.1 \%$ ), aves (XVIIc $=6 / 35$ o $17.1 \%$; XVIIa $=4 / 35$ o $11.4 ;$ XI=18/202 o $8.9 \%$; IV=32/209 o $15.3 \%$ ) y tortugas (IV=49/526 or $9.3 \%$ ) (ver Blasco et al. 2013a para más detalle).

Los grupos humanos que habitan Bolomor emplean la fracturación para acceder a la médula ósea. Esta actividad genera un número elevado de fragmentos de pequeñas dimensiones, que son la dinámica general de la secuencia, además de elementos diagnósticos que la caracterizan y la diferencian de la realizada por otros agentes. Así, se han registrado impactos de percusión, estigmas, contragolpes, conos de percusión, lascas medulares, esquirlas parásitas y fracturación por flexión o peeling. En total se han identificado evidencias de fracturación antrópica en 82 restos de ungulados en el subnivel XVIIc (6.3\%), 117 en el XVIIa (6.8\%), 57 en el nivel XI (5.4\%) y 839 en el IV (3.3\%). Los datos obtenidos a partir de la distribución de muescas de percusión han permitido observar cierta sistematización sobre zonas y partes anatómicas concretas (Blasco et al. 2013b). Por el contrario, no se han identificado elementos diagnósticos resultantes de la fracturación por percusión en huesos 


\begin{tabular}{|c|c|c|c|c|c|c|c|c|c|c|}
\hline & \multicolumn{4}{|l|}{ Aves } & \multicolumn{4}{|c|}{ Lagomorfos } & \multicolumn{2}{|c|}{ Tortugas } \\
\hline & XVIIc & XVIIa & XI & IV & XVIIc & XVIIa & XI & IV & XI & IV \\
\hline Cráneo & & & 13 & & & 33,3 & 71 & & & \\
\hline Maxilar & & & & & & 10 & 7,1 & & & \\
\hline Mandíbula & & & & & & 66,7 & 36 & 50 & & \\
\hline Fúrcula & & & 63 & & & & & & & \\
\hline Vértebra & 0,8 & 0,3 & 9,8 & & 5,4 & 6,4 & 6 & 3,9 & & 3,9 \\
\hline Costilla & & & 2,9 & & & 2,6 & 2,2 & 1 & & \\
\hline Pelvis & & & 25 & 10 & 47,2 & 73,3 & 43 & 43 & & 15,7 \\
\hline Escápula & 8,3 & & 25 & 23 & 25 & 46,7 & 57 & 33 & 50 & 21,1 \\
\hline Coracoides & 33,3 & 28,6 & 94 & 63 & & & & & & 2,6 \\
\hline Húmero & 50 & 42,9 & 75 & 33 & 41,7 & 60 & 64 & 50 & & 94,7 \\
\hline Radio & 8,3 & 7,1 & 56 & 40 & 54,2 & 66,7 & 71 & 40 & & 10,5 \\
\hline Ulna & 58,3 & 64,3 & 63 & 43 & 45,8 & 36,7 & 29 & 43 & & 2,6 \\
\hline Fémur & & 7,1 & 81 & 33 & & 53,3 & 43 & 28 & 50 & 73,7 \\
\hline Tibia & 8,3 & 7,1 & 88 & 47 & 83,3 & 76,7 & 50 & 45 & & 36,8 \\
\hline Fíbula & & & 6,2 & & & & & & & 10,5 \\
\hline Patella/Sesam. & & & & & 36,7 & 7,1 & & & & \\
\hline Carpal/Tarsal & & & & & 3,8 & & & & & \\
\hline Astrágalo & & & & & & 50 & 36 & 10 & & \\
\hline Calcáneo & & & & & & 46,7 & 21 & 75 & & \\
\hline $\mathrm{Mtc} / \mathrm{Cmc}$ & 50 & 35,7 & 69 & 40 & 12,5 & 9,2 & 16 & 31 & & \\
\hline $\mathrm{Mtt} / \mathrm{Tm} t$ & 33,3 & & 63 & 30 & 45,8 & 23,3 & 20 & 36 & & \\
\hline Metapodio & & & & & & & 11 & & & \\
\hline Falange & 5 & 0,6 & 14 & 13 & & 8,9 & 9,8 & 14 & & \\
\hline Caparazón & & & & & & & & & & 42,1 \\
\hline Plastron & & & & & & & & & 100 & 68,4 \\
\hline
\end{tabular}

Tabla 2. \%MAU para aves, lagomorfos y tortugas procedentes de la Cova del Bolomor. Mtc= metacarpo; $\mathrm{Mtt}=$ metatarso; $\mathrm{Cmc}=$ carpometacarpo; $\mathrm{Tmt}=$ tarsometatarso.

de lepóridos y aves. No obstante, las fracturas en este tipo de presas suele producirse cerca de las articulaciones de estilopodios y zeugopodios produciendo cilindros diafisarios (NISP XVIIc $=31$ of 132 estilopodios y zeugopodios; XI=15/138; IV=71/182) y articulaciones aisladas (NISP XVIIc=95/132; XI=50/138; IV=51/182). A partir de los criterios establecidos por Laroulandie (2005), Pérez Ripoll (2005), Landt (2007), Lloveras et al. (2009) y Sanchis Serra (2010), se han identificado mordiscos humanos sobre conejos, aves y tortugas. Estas alteraciones óseas en ocasiones configuran bordes crenulados y levantamientos pronunciados de la superficie cortical en los extremos de las fracturas (Blasco y Fernández Peris 2009, 2012a; Blasco et al. 2013a).

El conjunto faunístico de Bolomor también recoge modificaciones consecuentes de la termoalteración en el nivel XI ( $\mathrm{NR}=645 / 1047$ o $61.6 \%$ ) y en el IV (NR=15585/25323 o 61.5\%) con predominio de las vértebras y tibias en el nivel XI y fragmentos de huesos largos de tallas medias y pequeñas en el nivel IV (ver Blasco et al. 2013a para más detalle). Los grados de cremación en lagomorfos y aves se registran principalmente en las articulaciones de los huesos de las extremidades, y en el caso de las tortugas, en la cara dorsal de los caparazones (Blasco 2008).

Los homínidos, sin embargo, no son los únicos agentes que actúan sobre el registro faunístico de la Cova del Bolomor. Los carnívoros también intervienen sobre el conjunto dejando mordeduras y fracturas principalmente sobre el esqueleto apendicular proximal de tallas medias y grandes, que son, por otro lado, las predominantes de la secuencia. A pesar 

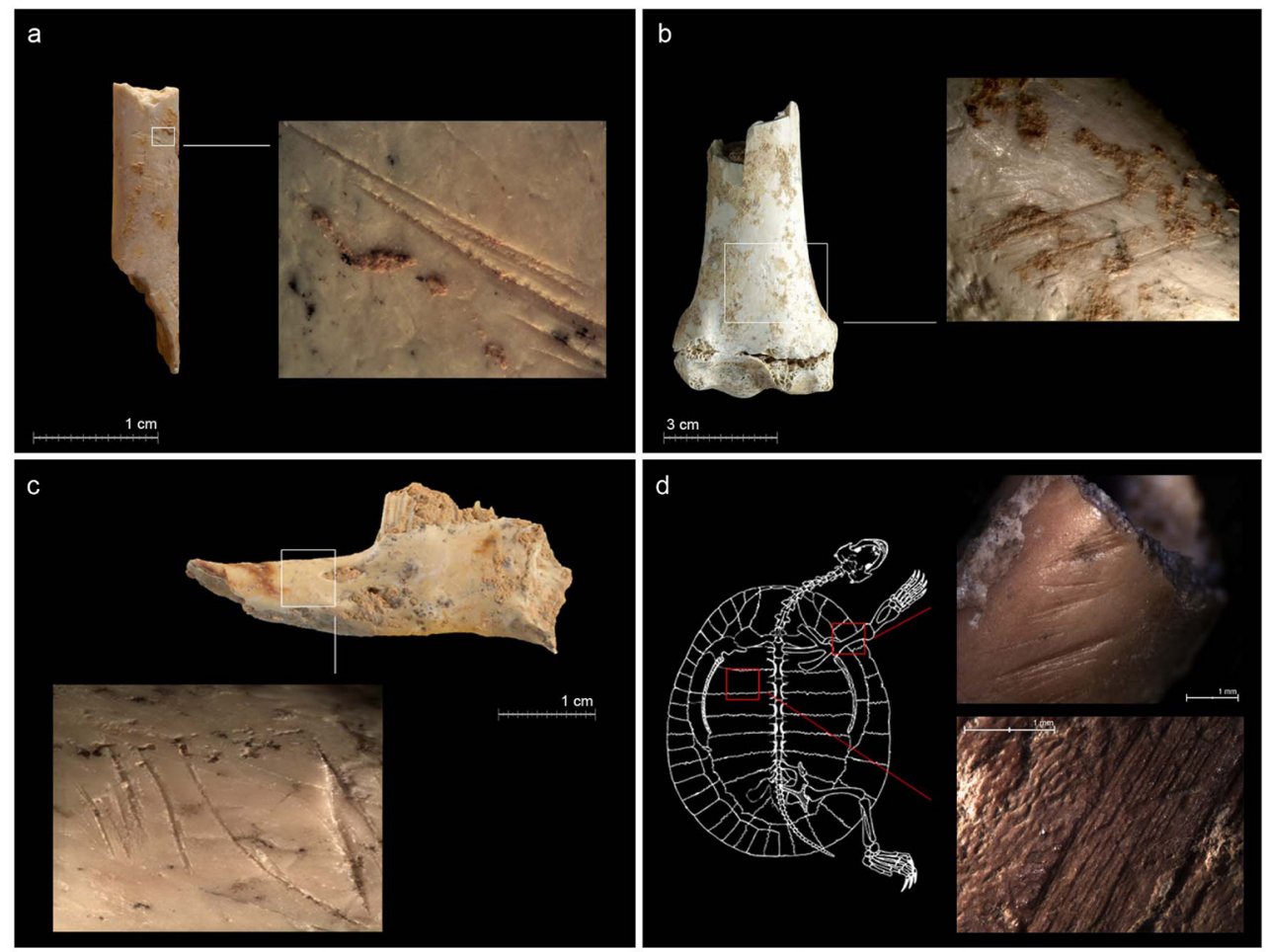

Figura 4. a) Marcas de corte sobre una tibia de conejo (O. cuniculus) del subnivel XVIIc; b) marcas de corte sobre un húmero de cisne (C. olor) del nivel XII; c) marcas de corte sobre una mandíbula de conejo (O. cuniculus) del subnivel XVIIc; d) marcas de corte sobre restos de tortuga (T. hermanni) del nivel IV. Extraído de Blasco y Fernández Peris (2012a).

de estas evidencias, la presencia de carnívoros es baja o casi ausente en algunos niveles (NR XVIIc $=53 / 1307$ o 4\%; XVIIa=102/1732 o $5.8 \%$; XI=8/1047 o 0.7\%; IV=142/25323 o 0.5\%). Los daños producidos por carnívoros sobre restos de pequeñas presas son también escasos, o incluso ausentes en el caso del nivel $\mathrm{XI}$, y corresponden principalmente a huesos con grados bajos de digestión (NISP XVIIc=24/500 o 4.8\%; XVIIa=74/659 o 11.2\%; IV=2/1832 o 0.1\%). Estas alteraciones podrían asociarse a actividades esporádicas de pequeños mamíferos carnívoros o rapaces (intrusiones naturales) (Blasco et al. 2013a, 2013b).

\section{Discusión}

Los homínidos del Pleistoceno medio europeo, y en particular los de Bolomor, parecen demostrar una elevada plasticidad, tanto en los sistemas de obtención de recursos, como en la utilización de un amplio espectro de presas (e.g., Blasco et al. 2010a; Blasco et al. 2013a; Hardy et al. 2013). Las características del entorno más inmediato parecen determinar en gran medida la representación taxonómica en el yacimiento, en el que la proximidad de la línea de costa hace que las fluctuaciones marinas queden reflejadas a nivel faunístico (ver Blasco 2011; Blasco et al. 2013a para más detalle). La situación propia del enclave parece ejercer también influencia sobre las primeras fases de la secuencia de aprovechamiento, en las que los animales con pesos superiores a los $20 \mathrm{~kg}$ suelen ser descuartizados y preparados para el transporte en el lugar de obtención (ver Blasco 2011; Blasco et al. 2013a para más detalle). 
No obstante, aquí deberíamos considerar que otros condicionantes podrían existir más allá del peso de las presas o la localización del yacimiento, como por ejemplo tradiciones culturales que lleven a una selección sistemática de las partes anatómicas. A pesar de esto, las limitaciones en función del peso parecen estar ampliamente reflejadas en algunos yacimientos interpretados como lugares de matanza al aire libre o trampas naturales (e.g. Scott 1980, 1986; Santonja et al. 1980; Callow y Conford 1986; Anzidei et al. 1989; Auguste 1995; Gaudzinski et al. 1996; Anzidei y Cerilli 2001; Villa et al. 2005), aunque es cierto que, en ocasiones, los homínidos podrían transportar porciones anatómicas diversas en función de las circunstancias inmediatas o variables tales como el número de porteadores, la distancia al lugar de hábitat, el momento del día, la tecnología disponible, etc. (Bunn and Kroll 1986; Bunn 1993; O'Connell et al. 1990; Gifford-González 1993; Faith et al. 2009). Esta diversidad comportamental también quedaría reflejada en el espectro de presas potenciales que los homínidos son capaces de explotar.

De forma concreta, la Cova del Bolomor evidencia un uso reiterado de pequeñas presas (lagomorfos, aves y en menor medida, quelonios) a lo largo de una secuencia de más de 250 kya, constituyendo un enclave excepcional para estudiar y valorar los posibles cambios de la dieta cárnica durante el Pleistoceno medio desde una perspectiva diacrónica. Las evidencias proporcionadas por Bolomor no parecen ajustarse a las respuestas propuestas desde el enfoque de la "Revolución de Amplio Espectro" -i.e., elementos como la densidad poblacional, la presión cinegética, la presión ambiental, la estabilidad territorial o el estadio tecnológico de desarrollo no parecen explicar el inicio temprano de este fenómeno en el enclave (Stiner et al. 2000; Stiner 2001; Stiner y Munro 2002; Bar-El y Tchernov 2001; Aura et al. 2002; Hockett y Haws 2002; Fontana 2003; Byers y Broughton 2004). En este sentido, podrían existir otros factores, como por ejemplo la tasa de encuentros vinculada al predominio de ciertos animales en el medio, que condicionen de manera más determinante la presencia de lepóridos, aves y quelonios en la dieta de los homínidos de Bolomor, y justifiquen por otro lado, la diversidad taxonómica observada en el yacimiento. Vinculado a esto, la dinámica ocupacional también podría influir en la representación taxonómica de las acumulaciones de origen antrópico, ya que una mayor estabilidad o permanencia en el enclave podría desembocar en una mayor diversidad específica. Este tipo de asentamientos no estarían dirigidos a la explotación de un taxón en concreto, tan propio de las ocupaciones estacionales en las que la existencia de una especie altamente predominante sobre el resto respondería a una caza selectiva, que constituiría a su vez el objetivo de la ocupación (ver Blasco 2011; Blasco et al. 2013a para más detalle). En este tipo de asentamientos, la representación específica parece mostrarse menos diversa y los taxones que no conforman el fin de la ocupación apenas tendrían representación (e.g., Monchot 1999; Lumley et al. 2004; Moncel et al. 2005; Moncel y Pathou-Mathis 2005; Moigne et al. 2006; Moncel et al. 2008). Sobre esta base, debemos tener en cuenta que la dieta humana, interpretada a partir de los conjuntos faunísticos, podría estar condicionada por múltiples factores, como el patrón ocupacional, los integrantes del grupo y los roles sociales que desarrollan dentro de él, la diversidad comportamental del propio individuo y/o grupo, y las propias características del medio circundante local. Desde esta perspectiva, es necesario tener en cuenta también que la existencia de variables no controladas podrían provocar la incorporación de ciertos recursos a la dieta. Estas variables no tendrían porqué estar siempre relacionadas con la necesidad de optimizar los recursos, sino que podrían enmarcarse dentro del enfoque de la ecología nutricional (Hockett y Haws 2002, 2009; Haws 2003; Hockett 2006, 2007) o responder a normas culturales o relaciones sociales que conllevasen algún tipo de retorno cultural, social o incluso simbólico al margen del puramente económico (Alhaique 1994; Bar-El y Tchernov 2001; Fontana 2003; Lupo y Schmitt 2005; Wadley 2010). A pesar 
de que estas posibilidades podrían haber existido, en el caso de Bolomor, las evidencias arqueológicas disponibles en este momento no permiten inferir la captura de lagomorfos, aves o quelonios para otro fin que no sea el comestible, como por ejemplo el tecnológico o, incluso el simbólico observado en otros yacimientos de cronología más temprana (e.g., Mourer-Chauviré 1989; Hockett 1994; Honeisen y Peyer 1994; Leesch 1997; Speth y Tchernov 2002; Laroulandie y D’Errico 2004; Fiore et al. 2004; Soressi et al. 2008; Gaudzinski y Niven 2009; Peresanni et al. 2011; Finalyson et al. 2012; Morin y Laurandie 2012). A pesar de ello y como ya fue apuntado en Blasco (2011), no podemos descartar la existencia de otro tipo de retorno cultural o social, que no quede evidenciado en el registro arqueológico y que se produjese de forma puntual o reiterada (Alhaique 1994; Bar-El y Tchernov 2001; Fontana 2003; Lupo y Schmitt 2005; Wadley 2010).

Algunos de los elementos usados comúnmente para explicar el cambio en la subsistencia a partir del Paleolítico superior en Europa y Próximo Oriente se han asociado subyacentemente con el "Comportamiento Humano Moderno" (Modern Human Behaviour -MHB). A nivel zooarqueológico, éste ha sido definido a partir de las capacidades para cazar grandes y peligrosos animales, la capacidad para hacer un uso intensivo de los recursos estacionales, y la capacidad para explotar pequeñas presas, especialmente aquellas de movimiento rápido -e.g., aves, lepóridos y especies nadadoras (peces) (e.g., Klein et al. 1987; Milo 1998; Marean et al. 2000; McBrearty y Brooks 2000; Clark y Plug 2008; Faith 2008). Aunque estas actividades se han relacionado frecuentemente con la presencia de Homo sapiens, varios de estos elementos utilizados por la Arqueología teórica parecen observarse en algunos conjuntos europeos del Pleistoceno medio e inicios del superior. Así, la capacidad de los grupos humanos de este periodo para enfrentarse con éxito a animales peligrosos, como grandes carnívoros, se ha documentado en algunos yacimientos, como por ejemplo en TD101 de Gran Dolina (Blasco et al. 2010b), Boxgrove (Etling 2003), Biache Saint Vast (Auguste 1995) o Tabun, Skhul y El-Wad (Marín 2011), y en cronologías más recientes como en Arcysur-Cure (David 1997), Prélétang (Tillet 2002), Sant Agostino (Stiner 1994), la Grotta delle Fate y la Madonna dell' Arma (Tillet 2002). La utilización de recursos con carácter estacional también se registra en enclaves europeos como Orgnac 3 (Moncel et al. 2005), Payre (Moncel y Pathou-Mathis 2005; Moncel et al. 2008) y en los niveles L, J y F de la Caune de l'Aragó (Monchot 1999; Lumley et al. 2004; Moigne et al. 2006). La obtención de animales pequeños de movimiento rápido o fast-moving small prey parece ser también un fenómeno presente en algunas localidades del Pleistoceno medio, bien como recurso puntual (Guennouni 2001; Costamagno y Laroulandie 2004; Roger 2004; Hardy y Moncel 2011) o bien, como fenómeno recurrente en yacimientos como Terra Amata C1b (Guennouni 2001; Valensí y Guennouni 2004), la Cova del Bolomor (Blasco 2008; Sanchis Serra y Fernández Peris 2008; Blasco y Fernández Peris 2009; Sanchis Serra 2010; Blasco y Fernández Peris 2012; Blasco 2011; Blasco et al. 2013a, 2013b), el nivel 4 de Canalettes (Cochard et al. 2012) y con una contribución mixta, el subnivel TD10-1 de Gran Dolina (Blasco 2011; Blasco et al. 2013a). Sin embargo, el tema más controvertido en estas cronologías reside en la inclusión del pescado en la dieta y en consecuencia, la práctica de actividades como la pesca. A pesar de ello, el aprovechamiento humano esporádico de recursos acuáticos (galápagos, cocodrilos, osteíctios, siluriformes, etc.) parece remontarse a cronologías del Pleistoceno inferior africano a partir de evidencias tafonómicas como marcas de corte (Braun et al. 2010; Archer y Braun, 2013; Archer et al. 2014), y extenderse por Asia, como sugieren los estudios ecológicos en hábitats costeros entre 1.5 y $0.9 \mathrm{Ma}$ en Indonesia (Joordens et al. 2009). Este panorama, unido a la inclusión temprana de pequeñas presas de movimiento rápido a la dieta humana en algunos enclaves arqueológicos, podría implicar que las transformaciones en los sistemas económicos no 
se desarrollaron de forma lineal dentro del marco temporal y espacial, sino que más bien estuvieron condicionados por la diversidad comportamental, los factores socio-culturales y el patrón ocupacional, así como por las características locales propias del medio donde los grupos humanos desarrollaron sus actividades (Blasco 2011; Cochard et al. 2012; Blasco et al. 2013a). Sobre esta base, los elementos faunísticos que definen la "modernidad" en el comportamiento humano podrían estar presentes desde momentos tempranos en Europa. Así, las ideas desarrolladas por McBrearty y Brooks (2000) sobre la adquisición progresiva y escalonada de este tipo de pautas en África durante la Middle Stone Age, podría también ser identificada entre los homínidos europeos. Desde esta perspectiva, "The revolution that wasn' $t$ ", podría no ser patrimonio exclusivo del linaje africano (los sapiens), sino que también podría ser aplicado al linaje europeo (los neandertales) (Blasco 2011:650).

\section{Conclusiones}

Los datos obtenidos han permitido documentar una diversidad relevante en el comportamiento de los grupos humanos de la Cova del Bolomor. Tales variaciones quedan reflejadas no sólo en el espectro de presas que los homínidos son capaces de explotar, sino también en la variedad de estrategias de subsistencia que son capaces de desarrollar. Dentro del espectro de presas potenciales, se ha documentado la obtención y procesamiento de pequeños animales. Este fenómeno no parece ajustarse a las respuestas propuestas para explicar la inclusión sistemática de pequeños animales a la dieta humana desde el enfoque de la BSR. Desde esta perspectiva, quizás la tasa de encuentros vinculada al predominio de ciertos animales en el medio, sea la que condicione de manera más determinante la presencia de pequeños animales, y justifique por otro lado, la diversidad taxonómica identificada. Junto a los factores ambientales, hay que sumar los socio-culturales y la propia dinámica ocupacional, la cual también parece condicionar la representación específica en las acumulaciones de origen antrópico. Sobre esta base, es posible plantear la existencia de numerosos cambios dietéticos cuya expresión más evidente parece localizarse en las transformaciones observadas por Binford (1968) y Flannery (1969) en la transición Pleistoceno-Holoceno. A partir de los datos proporcionados por Bolomor, los cambios en la dieta no parecen ser lineales en el marco temporal y espacial, sino que parecen estar condicionados por factores diversos, como la propia diversidad comportamental, el patrón ocupacional y las características locales del medio donde se desenvuelven los diferentes grupos humanos del territorio europeo durante el Pleistoceno medio y el inicio del superior.

Agradecimientos: La excavación de la Cova del Bolomor forma parte del programa de excavaciones arqueológicas del Servicio de Investigación Prehistórica (SIP) del Museo de Prehistoria de Valencia bajo la autoridad provincial de la Diputación de Valencia (España). Esta investigación es apoyada por financiación procedente del Ministerio español de Ciencia e Innovación con número de proyecto CGL-BOS-2012-34717. Ruth Blasco es receptora de una beca post-doctoral Beatriu de Pinós-A de la Generalitat de Catalunya co-financiada por la Unión Europea mediante las Acciones Marie Curie, FP7.

\section{Bibliografía citada}

Acuña, R.

1982-1987 Relaciones geográficas del siglo XVI. UNAM, México.

Alhaique, F.

1994 Taphonomic analysis of the faunal remains from the " $\mathrm{P}$ " and " $\mathrm{M}$ " layers of the Arene Candide (Savona, Italy). Quaternaria Nova IV: 263-295. 
Anzidei, A.P; Angelelli, R; Arnoldus-Huyzendveld

1989 Le gisement pleistocene de 'La Polledrara' di Cecanibbio (Rome, Italy) L'Anthropologie 93: 749-782.

Anzidei, A. P., Cerilli, E.

2001 The fauna of La Polledrara di Cecanibbio and Rebibbia-Casal de' Pazzi (Rome, Italy) as an indicator for site formation processes in a fluvial environment. The World of Elephants (ed. por G. Cavarretta, P. Gioia, M. Mussi, M.R Palombo), pp. 167-171. Proceedings of the First International Congress. Consiglio Nazionale delle Ricerche, Rome.

Archer, W.; Braun, D.R.

2013 Investigating the Signature of Aquatic Resource Use within Pleistocene Hominin Dietary Adaptations. PLoS ONE 8(8): e69899

Archer, W.; Braun, D.R.; Harris, W.K.J.; McCoy, J.T.; Richmond, B.G.

2014 Early Pleistocene aquatic resource use in the Turkana Basin. Journal of Human Evolution http://dx.doi.org/10.1016/j.jhevol.2014.02.012

Arsuaga, J.L., Fernández Peris, J., Gracia-Téllez, A., et al.

2012 Fossil human remains from Bolomor Cave (Valencia, Spain). Journal of Human Evolution 62: 629-639.

Auguste, $\mathrm{P}$.

1995 Chasse et charognage au Paléolithique moyen : l'apport du gisement de Biache-SaintVaast (Pas-de-Calais). Bull. Soc. Prehist. Fr. 92 : 155-167.

Aura, J.E.; Villaverde, V.; Pérez Ripoll, M.; Martínez Valle, R. y Guillem, P.M.

2002 Big game and small prey: Paleolithic and Epipaleolithic economy from Valencia (Spain). Journal of Archaeological Method and Theory 9 (3): 215-267.

Azorit, C.; Analla; M.; Carrasco; R.; Calvo, J.A. and Múñoz Cobo, J.

2002 Teeth eruption pattern in red deer (Cervus elaphus hispanicus) in southern Spain. Anales de Biología, 24: 107-114.

Bar-El, T. and Tchernov, E.

2001 Lagomorph remains at prehistoric sites in Israel and Southern Sinai. Paléorient 26: 93-109.

Binford, L.R.

1968 Post-Pleistocene Adaptations. New Perspectives in Archaeology (ed. por S.R. Binford, and L.R. Binford), pp. 313-341. Aldine, Chicago.

1981 Bones: Ancient Men and Modern Myths. Academic Press, New York.

Blasco, R.

2008 Human consumption of tortoises at Level IV of Bolomor Cave (Valencia, Spain). Journal of Archaeological Science 35: 2839-2848.

2011 La amplitud de la dieta cárnica en el Pleistoceno medio peninsular: una aproximación a partir de la Cova del Bolomor (Tavernes de la Valldigna, Valencia) y del subnivel TD10-1 de Gran Dolina (Sierra de Atapuerca, Burgos). Ph. dissertation. Universitat Rovira i Virgili, Tarragona. 
Blasco, R. y Fernández Peris, J.

2009 Middle Pleistocene bird consumption at level XII of Bolomor Cave (Valencia, Spain). Journal of Archaeological Science 36: 2213-2223.

2012a A uniquely broad spectrum diet during the Middle Pleistocene at Bolomor Cave (Valencia, Spain). Quaternary International 252: 16-31.

2012b Small and large game: human use of diverse faunal resources at Level IV of Bolomor Cave (Valencia, Spain). C. R. Palevol 11: 265-282.

Blasco, R., Fernández Peris, J. y Rosell, J.

2010a Several different strategies for obtaining animal resources in the late Middle Pleistocene: the case of level XII at Bolomor Cave (Valencia, Spain). C. R. Palevol 9: 171-184.

Blasco, R.; Rosell, J.; Arsuaga, J.L.; Bermúdez y J.M and Carbonell, E.

2010b The hunted hunter: the capture of a lion (Panthera leo fossilis) at the Gran Dolina site (Sierra de Atapuerca, Burgos, Spain). Journal of Archaeological Science 37: 2051-2060

Blasco, R., Rosell, J. y Fernández Peris, J.

2013a Environmental availability, behavioural diversity and diet: a zooarchaeological approach from the TD10-1 sublevel of Gran Dolina (Sierra de Atapuerca, Burgos, Spain) and Bolomor Cave (Valencia, Spain). Quaternary Science Reviews 70: 124-144.

Blasco, R., Rosell, J. y Domínguez-Rodrigo, M.

2013b. Learning by heart: cultural patterns in the faunal processing sequence during the Middle Pleistocene. PLoS ONE 8 (2), e55863.

Blumenschine, R. J. y Selvaggio, M.

1988 Percussion marks on bone surfaces as a new diagnostic of hominid behavior. Nature, 333: 763-765.

Braun, D.R; Harris, J.W.K. y Levin, N.E.

2010 Early hominin diet included diverse terrestrial and aquatic animals 1.95 Ma in East Turkana, Kenya. Proc. Natl. Acad. Sci. USA 107(22), 10002-10007.

Bunn, H.T.

1993 Bone assemblages at base camps: a further consideration of carcass transport and bone destruction by the Hadza. In: Hudson, J. (Ed.), From Bones to Behavior. Ethnoarchaelogical and Experimental Contributions to the Interpretation of Faunal Remains. Center for Archaeological Investigations. Southern Illinois University at Carbondale, pp. 156-168.

Bunn, H.T. y Kroll, E.M.

1986 Systematic butchery by Plio-Pleistocene hominids at Olduvai Gorge, Tanzania. Current Anthropology 27: 431-452.

Byers, D.A., Broughton, J.M.

2004 Holocene environmental change, artiodactyl abundances, and human hunting strategies in the Great Basin. American Antiquity 69: 235-256.

Callow, P. y Conford, J.M. (eds.)

1986 La Cotte de Saint-Brelade, 1961-1978. Geo Books. Norwich. 
Capaldo, S.D. y Blumenschine R.J.

1994 A quantitative diagnosis of notches made by hammerstone percussion and carnivore gnawing in bovid long bones. American Antiquity 59: 724-748.

Clark, J.L. y Plug, I.,

2008 Animal exploitation stategies during the South African Middle Stone Age: Howiesons Poort and post-Howiesons Poort fauna from Sibudu Cave. Journal of Human Evolution 54: 886-898.

Cochard, D., Brugal, J.-Ph., Morin, E. y Meignen, L.

2012 Evidence of small fast game exploitation in the Middle Paleolithic of Les Canalettes (Aveyron, France). Quaternary International 264 : 32-51.

Costamagno, S., Laroulandie, V.

2004 L'exploitation des petits vertébrés dans les Pyrénées françaises du Paléolithique au Mésolithique : un inventaire taphonomique et archéozoologique. Petits Animaux et Sociétés Humaines Du Complement Alimentaire aux Ressources Utilitaires (ed. por J.-P. Brugal, J. Desse), pp. 403-416. XXIV Rencontres Internationales d'Archéologie et d'Histoire d'Antibes, Editions APDCA, Antibes.

Cuartero, F.

2008 Tecnología lítica en la Cova del Bolomor IV: ¿una economía de reciclado? Saguntum 39: $27-44$

David, F.

1997 Les ours du Châtelperronien de la Grotte du Rennea Arcy-sur-Cure (Yonne). L'Ours et l'Homme (ed. por T. H. Tillet, L.R. Binford), pp.185-192. Actes du Colloque d'Auberives-enRoyans, vol. 100. Eraul, Liège.

Etling, $\mathrm{K}$.

2003 Hunting bears: black, brown, grizzly, and polar bears. Woods N’Water, Inc., Bellvale (USA)

Faith, J.T.

2008 Eland, buffalo, and wild pigs: were Middle Stone Age humans ineffective hunters? Journal of Human Evolution 55: 24-36.

Faith, J.T.; Domínguez-Rodrigo, M. y Gordon, A.D.

2009 Long-distance carcass transport at Olduvai Gorge? A quantitative examination of Bed I skeletal element abundances. Journal of Human Evolution 56: 247-256.

Fernández Peris, J.

2007 La Cova del Bolomor (Tavernes de la Valldigna, Valencia). Las industrias líticas del Pleistoceno medio en el ámbito del Mediterráneo peninsular. Serie de Trabajos Varios del SIP (Servicio de Investigación Prehistórica), Valencia 108.

Fernández Peris, J., Barciela González, V., Blasco, R. y Cuartero, F., Sañudo, P.

2008 El Paleolítico Medio en el territorio valenciano y la variabilidad tecno-económica de la Cova del Bolomor. Treballs d'Arqueologia 14: 141-169. 
Fernández Peris, J., Barciela, V. y Blasco, R.

2012 The earliest evidence of hearths in southern Europe: the case of Bolomor Cave (Valencia, Spain). Quaternary International 247: 267-277.

Finlayson, C., Brown, K., Blasco, R., Rosell, J., Negro, J.J. et al.

2012 Birds of a feather: Neanderthal exploitation of raptors and corvids. PLoS One 7 (9): e45927.

Fiore, I., Gala, M. and Tagliacozzo, A.

2004 Ecology and subsistence strategies in the eastern Italian Alps during the Middle Paleolithic. International Journal of Osteoarchaeology 14: 273-286.

Flannery, K.

1969 Origins and ecological effects of early domestication in Iran and the Near East. The Domestication and Exploitation of Plants and Animals (ed. por P.J. Ucko, G.W. Dimbleby), pp. 73-100. Chicago.

Fumanal, M.P.

1993 El yacimiento premusteriense de la Cova del Bolomor (Tavernes de la Valldigna, País Valenciano). Cuadernos de Geografía 54: 223-248.

Gaudzinski, S., Bittmann, F., Boenigk, W., Frechenc, M., Van Kolfschoten, T. 1996 Palaeoecology and Archaeology of the Kärlich-Seeufer open-air site (Middle Pleistocene) in the Central Rhineland, Germany. Quaternary Research 46(3): 319-334.

Gaudzinski-Windheuser, S. and Niven, L.

2009 Hominin subsistence patterns during the Middle and Late Pleistocene in northwestern Europe. The Evolution of Hominin Diets: Integrating Approaches to the Study of Palaeolithic Subsistence (ed. por J.J. Hublin, M.P. Richards), pp. 99-111. Springer Verlag, Berlin.

Gifford-Gonzalez, D.

1993 Gaps in the zooarchaeological analyses of butchery: is gender an issue? In: Hudson, J. (Ed.), From Bones to Behavior. Ethnoarchaelogical and Experimental Contributions to the Interpretation of Faunal Remains. Center for Archaeological Investigations. Southern Illinois University at Carbondale, pp. 181-199.

Guennouni, K.

2001 Les lapins du Pléistocène moyen et supérieur de quelques sites préhistoriques de l'Europe méditerranéenne: Terra-Amata, Orgnac 3, Baume Bonne, la Grotte du Lazaret, la Grotte du Boquete de Zafarraya, Arma delle Manie. Etude paléontologique, archéozoologique et taphonomique. Museum National d'Histoire Naturelle, Paris.

Hardy, B.L., y M.-H. Moncel

2011 Neanderthal Use of Fish, Mammals, Birds, Starchy Plants and Wood 125-250,000 Years Ago. PLoS ONE 6(8), e23768

Hardy, B.L.; Moncel, M.-H.; Daujeard, C. et al.

2013 Impossible Neanderthals? Making string, throwing projectiles and catching small game during Marine Isotope Stage 4 (Abri du Maras, France). Quaternary Science Reviews 82: 23-40. 
Haws, J.A.

2003 An investigation of the later upper Paleolithic and Epipaleolithic hunter-gatherer subsistence and settlement patterns in central Portugal. Ph. Dissertation, University of Wisconsin-Madison.

Hockett, B.S.

1994 A descriptive reanalysis of the leporid bones from Hogup cave, Utah. Journal of California and Great Basin. Anthropology 16: 106-117.

Hockett, B.S.

2006 Climate, dietary choice, and the Paleolithic hunting of rabbits in Portugal. Animais na pré-historia e arqueología da península Ibérica, p. 137-144. Actas do IV Congresso de arqueología peninsular, Universidade do Algarve, Faro.

Hockett, B.S.

2007 Small faunal use during the Middle and late Pleistocene of Portugal: a nutritional ecology perspective. From the Mediterranean Basin to the Portuguese Atlantic Shore: Papers in Honor of Anthony Marks (ed. por N.F. Bicho), pp. 29-35. Promontoria Monografica 07, Universidade do Algarve, Faro.

Hockett, B.S. y J.A. Haws

2002 Taphonomic and methodological perspectives of leporid hunting during the upper Paleolithic of the western Mediterranean basin. Journal of Archaeological Method and Theory 9 (3): 269-301.

Hockett, B.S. y J.A. Haws

2009 Continuity in animal resource diversity in the Late Pleistocene human diet of central Portugal. Before Farming 2: 1-14.

Honeisen, M. y S.Peyer

1994. Schweizerbild ein Jägerlager der Späteiszeit, beuträge und Dakumente zur Ausgrabung vor 100 jarhren. Vol. 2: Schaffhauser Archäologie.

Joordens J.C.A, Wesselingh F.P., Vos J.de, Vonhof H.B., Kroon D.

2009. Relevance of aquatic environments for hominins: a case study from Trinil (Java, Indonesia). Journal of Human Evolution 57: 656-671.

Klein, R.G., Nitecki, M.H., Nitecki, D.V.

1987 Reconstructing how early people exploited animals: problems and prospects. The Evolution of Human Hunting (ed. por M.H. Nitecki, D.V. Nietcki), pp. 11-45, Plenum Press, New York.

Landt, M. J.

2007 Tooth marks and human consumption: ethnoarchaeological mastication research among foragers of the Central African Republic. Journal of Archaeological Science 34: 1629-1640.

Laroulandie, $\mathrm{V}$.

2005. Bird Exploitation Pattern : the Case of Ptarmigan Lagopus sp. in the Upper Magdalenian Site of La Vache (Ariège, France). Feathers, grit and symbolism. Birds and humans in the ancient Old and New Worlds (ed. por G. Grupe, J. Peters), pp. 165-178. Proceedings of the 5th Meeting of the ICAZ Bird Working Group, Munich, 26-28 July 2004. Rahden/Westf : Verlag Marie Leidorf. 
Laroulandie, V. y F.d'Errico

2004 Worked bones from Buran-Kaya III level C and their taphonomic context. The Paleolithic of Crimea, III. The Middle Paleolithic and Early Upper Paleolithic of Eastern Crimea (ed. por V. Chabai, K. Monigal, A. Marks), pp. 37-48, ERAUL.

Leesch, D.

1997 Un campement magdalénien au bord du lac de Neuchatel. Contexte, vestiges, activités (ed. por P. Morel, W. Muller). Archéologie Neuchateloise, 19.

Lloveras, Ll.; Moreno-García, M. y Nadal, J.

2009. Butchery, coocking and human consumption marks on rabbit (Oryctolagus cuniculus) bones: an experimental study. Journal of Taphonomy 7: 179-201.

Lumley, H. d., Grégoire, S. y Barsky, D.

2004. Habitat et mode de vie des chasseurs paléolithiques de la Caune de l'Arago (600 000-400 000 ans). l'Anthropologie 108 : 159-184.

Lupo, K.D. y D.N. Schmitt

2005 Small prey hunting technology and zooarchaeological measures of taxonomic diversity and abundance: ethnoarchaeological evidence from Central African forest foragers. Journal of Anthropological Archaeology 24: 335-353.

Lyman, R.L.

2008. Quantitative Paleozoology. Cambridge University Press, New York.

Maguire, J.M.; Pemberton, D. and Collett, M.H.

1980 The Makapansgat limeworks grey breccia: hominids, hyaenas, hystricids or hillwash. Paleontologia africana 23: 75-98.

Marean, C.W., Abe, Y., Frey, C.J., Randall, R.C.

2000 Zooarchaeological and taphonomic analysis of the Die Kelders Cave 1 layers 10 and 11 Middle Stone Age larger mammal fauna. Journal of Human Evolution 38: 197-233.

Marín, A.B.

2011 Palaeolithic human subsistence in Mount Carmel (Israel). A taphonomic assessment of Middle and Early Upper Palaeolithic faunal remains from Tabun, Skhul and el-Wad. International Journal of Osteoarchaeology 23: 254-273.

McBrearty, S. y A. Brooks

2000 The Revolution That Wasn't: a New Interpretation of the Origin of Modern Human Behavior. Journal of Human Evolution 39: 453-563.

Milo, R.G.

1998. Evidence for hominid predation at Klasies River Mouth, South Africa, and its implications for the behavior of early modern humans. Journal of Archaeological Science 25: 99-133. 
Moncel, M.-H. y M. Patou-Mathis

2005 Site de Payre (Ardèche): les différentes occupations humaines dans leur contexte biochronologique et paléoclimatique. Données récentes sur les modalités de peuplement et sur le cadre chronostratigraphique, géologique et paléoanthropologique des industries du Paléolithique inférieur et moyen en Europe (ed. por N. Molines, M.-H. Moncel, J.L. Monnier), pp. 159-173. British Archaeological Reports S1364, Oxford

Moncel, M. H., Moigne, A. M. y Combier, J.

2005 Pre-Neandertal behaviour during isotopic stage 9 and the beginning of stage 8 . New data concerning fauna and lithics in the different occupation levels of Orgnac 3 (Ardèche, South-East France): occupation types. Journal of Archaeological Science 32: 1283-1301.

Moncel, M.-H., Auguste, P. y Ayliffe, L.

2008 Le site de Payre. Occupations humaines dans la vallée du Rhône à la fin du Pléistocène moyen et au début du Pléistocène supérieur. Mémoire de la Societé Préhistorique Française XLVI, Paris,

Monchot, $\mathrm{H}$.

1999 Mixture analysis and mammalian sex ratio among Middle Pleistocene mouflon of Arago Cave, France. Quaternary Research 52: 259-258.

Moigne, A. M., Palombo, M. R. y Belda, V.

2006 Les faunes de grands mammifères de la Caune de l'Arago (Tautavel) dans le cadre biochronologique des faunes du Pléistocène moyen italien. L'Anthropologie 110: 788-831.

Morin, E.

2012 Reassessing Paleolithic subsistence: the Neandertal and modern human foragers of Saint-Césaire. Cambridge University Press, Cambridge.

Morin, E. y V. Laroulandie,

2012 Presumed symbolic use of diurnal raptors by Neanderthals. PLoS ONE 7: e32856.

O'Connell, J.F.; Hawkes, K.; Jones, N.B.

1990 Reanalysis of large mammal body part transport among the Hadza. Journal of Archaeological Science 17: 301-316.

Peresani, M., Fiore, I., Gala, M., Romandini, M., Tagliacozzo, A.

2011 Late Neandertals and the intentional removal of feathers as evidenced from bird bone taphonomy at Fumane Cave 44 ky B.P., Italy. PNAS 108: 3888-3893.

Pérez Ripoll, M.

2005 Caracterización de las fracturas antrópicas y sus tipologías en huesos de conejo procedentes de los niveles gravetienses de la Cova de les Cendres (Alicante). Munibe 57: 239-254.

Pickering, T. y C.P. Egeland

2006 Experimental patterns of hammerstone percussion damage on bones: implications for inferences of carcass processing by humans. Journal of Human Evolution 33: 459-469. 
Roger, T.

2004 L'avifaune du Pléistocène moyen et supérieur du bord de la Méditerranée européenne: Orgnac 3, Lazaret (France), Caverne delle Fate, Arma delle Manie (Italie), Kalamakia (Grèce), Karain E (Turquie). Paléontologie, Taphonomie et Paléoécologie. Ph Dissertation, Institut de Paléontologie Humaine. Muséum National d'Histoire Naturel, Paris.

Romandini M, Peresani M, Laroulandie V, Metz L, Pastoors A, et al.

2014 Convergent Evidence of Eagle Talons Used by Late Neanderthals in Europe: A Further Assessment on Symbolism. PLoS ONE 9(7): e101278.

Sanchis Serra, A. y J. Fernández Peris

2008 Procesado y consumo antrópico de conejo en la Cova del Bolomor (Tavernes de la Valldigna, Valencia). Complutum 19(1): 25-46.

Sanchis Serra, A.

2010 Los lagomorfos del Paleolitico medio de la región central y sudoriental del Mediterráneo Ibérico. Caracterización tafonómica y taxonómica. Ph. Dissertation, Dpt. Arqueología y Prehistoria. Valencia, Universitat de València.

Santonja, M.; López Martínez, N. y Pérez González, A.

1980. Ocupaciones Achelenses en el Valle del Jarama (Arganda, Madrid). Diputación Provincial de Madrid, Madrid.

Scott, K.

1980 Two Hunting Episodes of Middle Palaeolithic Age at La Cotte de Saint-Brelade. Jersey (Channel Islands). World Archaeology 12 (2): 137-152.

Scott, K.

1986 The bone assemblages of layers 3 and 6. La Cotte de St Brelade (ed.por P. Callow, J. M. Cornford), pp. 159-183. Geo Books, Norwich.

Shipman, P. y J. Rose

1983 Early hominid hunting, butchering and carcass-processing behaviors: approches to the fossil record. Journal of Anthropological Archaeology 2: 57-98.

Shipman, P.; Foster, G. y Schoeninger, M.

1984 Burnt Bones and Teeth: an Experimental Study of Color, Morphology, Crystal Structure and Shrinkage. Journal of Archaeological Science 11: 307-25.

Simms, S. R.

1987 Behavioral ecology and huntergatherer foraging. AN exemple from the Great Bassin. BAR International Series 381,-103.

Soressi, M., Rendu, W., Texier, J.-P. et al.

2008. Pech-del' Azé I (Dordogne, France): Nouveau regard sur un gisement moustérien de tradition acheuléenne connu depuis le XIXe siècle. Les sociétés Paléolithiques d'un grand Sud-Ouest: Nouveaux gisements, nouvelles méthodes, nouveaux résultats (ed. por J.J.Jaubert, G. Bordesand, I. Ortega), pp. 95-132. Mémoire de la Société Préhistorique Française. 
Silver, I.A.

1969 La determinación de la edad en los animales domésticos. Ciencia en Arqueología: 229-239.

Speth, J.D. y E.Tchernov

2002 Middle Paleolithic tortoise use at Kebara Cave (Israel). Journal of Archaeological Science 29: 471-483.

Stiner, M.C.

1994 Honor among thieves: A zooarchaeological study of Neandertal ecology. Princeton University Press, Princeton

Stiner, M.C.

2001 Thirty years on the "Broad Spectrum Revolution" and paleolithic demography. PNAS 19: 6993-6996.

Stiner, M.C.; Weiner, S.; Bar-Yosef, O., Kuhn, S.L.

1995. Differential Burning, Recrystallization and Fragmentation of Archaeological Bone. Journal of Archaeological Science 22: 223-37.

Stiner, M.C., Munro, N.D., Surovell, T.A., Tchernov, E., Bar-Yolsef, O.

1999. Paleolithic population growth pulses evidenced by small animal exploitation. Science 283:190-194.

Stiner M.C., Munro N.D. y Surovell T.A.

2000 The tortoise and the hare. Small game use, the broad-spectrum revolution, and Paleolithic demography. Current Anthropoogy. 41: 39-73.

Stiner, M.C. and N.D. Munro

2002 Approaches to prehistoric diet breadth, demography, and prey ranking systems in time and space. Journal of archeological method and theory 9: 181-214.

Tillet, $\mathrm{T}$.

2002. Les grottes à ours et occupations néandertaliennes dans l'arc alpin et jurassien. L'Ours et l'Homme (ed. por T. Tillet, L.R. Binford), pp.167-184. Actes du Colloque d'Auberives en Royans, 1997, vol. 100. Eraul, Liège.

Valensí, P. y Guennouni, K.

2004 Comportements de subsistance et structures d'habitat sur le site de plein air de Terra Amata (Paléolitique inférieur, France). BAR International Series: 75-85.

Villa, P., Soto, E., Santonja, M., et al.

2005. New data from Ambrona: closing the hunting versus scavenging debate. Quaternary International 126-128: 223-250.

Wadley, L.

2010. Were snares and traps used in the Middle Stone Age and does it matter? A review and a case study from Sibudu, South Africa. Journal of Human Evolution 58: 179-192.

Winterhalder, B.

1981. Foraging strategies in the boreal forest: an analysis of Cree hunting and gathering. Huntergatherer foraging strategies (ed. por B. Winterhalder, E. Alden Smith), pp. 66-98. The University of Chicago Press, Chicago. 